\title{
THE VIRTUAL VEHICLE AND AUTOSAR - THE WAY FORWARD
}

How do you reuse embedded systems across vehicle platforms and programs? How do you minimize the barriers between functional domains and validate complex electro-mechanical systems early in the design phase?

For the last two decades automotive companies have worked tirelessly to optimize their new product creation processes. In the context of mechanical design it is now common practice for complete vehicles to be virtually modeled. Using 3D design tools such as CATIA from Dassault Systems, 3D geometrical parts are assembled and simulated as complete virtual vehicles in 3D Digital Mockups (DMU).

Automotive companies want to extend this concept to encompass the integration of electrical and electronic systems, including the systems architecture and embedded software. They want to simulate the control and interaction of embedded systems with the geometry of the vehicle and the environment it is interacting with. This 'intelligent' Digital Mockup is called a 'Functional Mockup'. The challenge is how to extend this approach to encompass embedded systems development, and the reuse of these systems, across different vehicle models, product lines and automotive brands.

\section{AUTOSAR - THE FOUNDATION FOR PRODUCT LINE ENGINEERING}

The first step to achieving the reuse of embedded systems is the adoption of the AUTOSAR standard, which serves as a platform for implementing systems in a way that greatly simplifies their reuse. AUTOSAR facilitates the decoupling of embedded applications from how they are physically implemented on the target Electronic Control Units (ECUs) that they run on. AUTOSAR Builder ${ }^{\odot}$ from Dassault Systèmes, provides an open, flexible and powerful authoring and simulation toolset that enables the rapid modeling, definition, simulation and deployment of embedded systems to target ECUs.

\section{VIRTUAL VEHICLE SIMULATION}

AUTOSAR Builder provides a simulation platform that facilitates Software-In-the-Loop testing of embedded systems at both the Virtual Functional Bus and ECU virtual prototyping levels. However, to scale to the complete system or vehicle level, it is essential to be able to compose a number of sub-systems in a single interoperable simulation environment. These sub-system models can come from many different tools including the Modelica based Dymola (Dassault Systèmes), ASCET (ETAS) or MATLAB/Simulink (The MathWorks).

The concept of a 'Functional Mockup Interface' (FMI) was defined by the European ITEA2 Modelisar project, whose goal was to improve the design of systems and embedded software in vehicles. FMI is an open, general and vendor independent tool interface standard that provides advanced runtime interoperability inter-

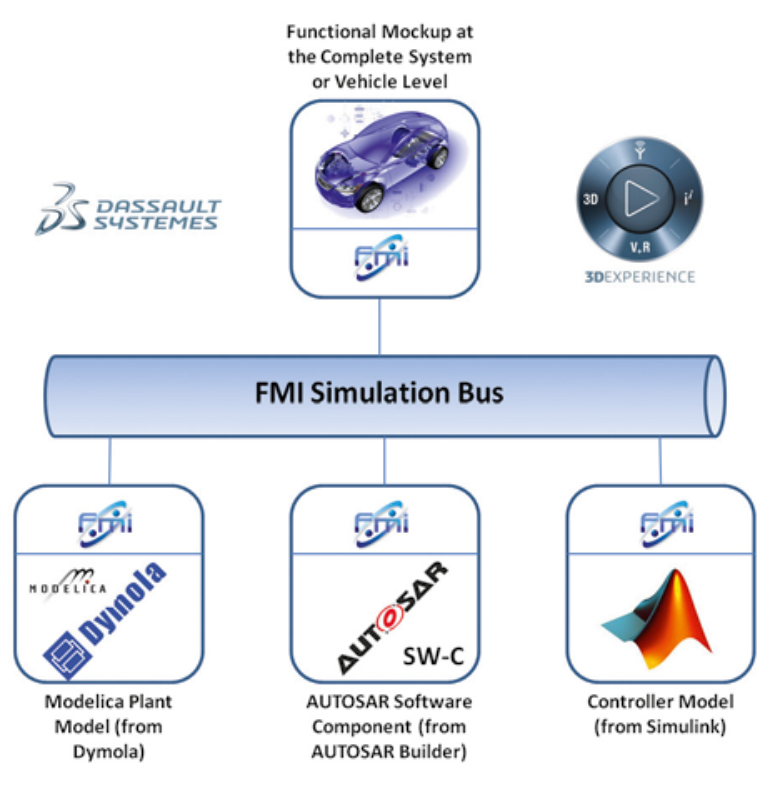

faces. These interfaces enable accurate system model compositions to be created by allowing several pre-compiled simulation units to be combined into one Functional Mockup simulation framework. Both AUTOSAR Builder and DYMOLA fully support FMI, enabling complex plant and ECU interactions to be validated using multilevel simulation approaches (e.g. SIL, MIL, HIL \& PIL).

\section{REUSABLE ARCHITECTURE - A HOLISTIC APPROACH}

Such a solution demands an integrated approach to the development, simulation and validation of integrated electrical, software and mechanical vehicle systems, through the design, decomposition and reuse of complex embedded systems using a multi-level approach that encompasses:

- Customer \& technical functions

- Function \& software nets

- Hardware topology

- Software partitioning (topology and interface definition)

- Embedded system architecture simulation

Dassault Systèmes 3DEXPERIENCE platform delivers a collaborative integrated development and co-simulation environment, leveraging AUTOSAR Builder, Dymola, CATIA, FMI \& Reqtify (for traceability of system requirements), on a rich platform with full lifecycle, data, configuration, IP rights, governance, project and program management capabilities.

For more information, connect to 3ds.com/AUTOSAR-Builder 